\title{
¿Facilitan los proyectos institucionales de participación la capacitación política?
}

\author{
María Jesús FunES \\ Universidad Nacional de Educación a Distancia (UNED) \\ mfunes@poli.uned.es
}

Recibido: 25-02-2015

Aceptado: 08-02-2016

\begin{abstract}
Resumen
La participación parte habitualmente de las bases sociales, organizadas en acciones colectivas más o menos duraderas y con desigual capacidad de influencia. Sin embargo, en las últimas décadas las instituciones políticas han puesto en marcha unos proyectos con el objetivo de implicar a la ciudadanía y de profundizar los hábitos democráticos de individuos y administraciones. Este artículo analiza el potencial de capacitación política de estos proyectos institucionales: si haber formado parte de los mismos cualifica como ciudadano activo, si aporta motivación hacia lo público, profundización (o creación) de voluntad política y habilidades para ejercerla. Se utilizan datos de un análisis comparado de cinco proyectos participativos en España y Francia lo que permite identificar qué proyectos contribuyen a la formación de sujetos políticos y de qué modo. Finalmente, para comprender mejor esta dimensión formativa, se la compara con la de otras formas participativas que desarrollan los individuos de manera espontánea.
\end{abstract}

Palabras clave: proyectos institucionales de participación; sujeto político; impactos educativos; empoderamiento; motivación política; voluntad política; habilidades participativas.

\section{Do Institutional Participatory Projects Contribute to the Development of Political Capabilities?}

\begin{abstract}
Participation usually sets off from the bottom up, taking the form of more or less enduring forms of collective action with varying degrees of influence. However, a number of projects have been launched by political institutions in the last decades with a view to engaging citizens in public affairs and developing their democratic habits, as well as those of the administration. This paper analyses the political qualifying capacity of the said projects, i.e. whether participating in them qualifies individuals to behave as active citizens; whether these projects foster greater orientation towards public matters, intensify (or create) political will, and provide the necessary skills and expertise to master this will. To answer these questions, data from the comparative analysis of five participatory projects in France and Spain are used, shedding light on which features of these participatory projects contribute to the formation of political subjects and in which way. Finally, in order to better understand this formative dimension, the formative capacity of institutional projects is compared with the formative dimension of other forms of participation spontaneously developed by citizens.
\end{abstract}

Key words: institutional participatory projects; political subject; educational impacts; empowerment; political motivation; political will; participation skills.

\section{Referencia normalizada}

Funes, M.J. (2016): “¿Facilitan los proyectos institucionales de participación la capacitación política?”, Politica y Sociedad, 53 (1), pp. 55-77.

Sumario: Introducción. 1. ¿Pretenden/pueden los proyectos institucionales de participación formar sujetos políticos? 2. ¿Qué queremos saber? 3. Revisando lo que ya sabemos, ...y yendo más allá. 4. ...Y 
yendo más allá: ¿construyen sujetos políticos? 5. Comparando con otras formas de construir ciudadanos. 6. Conclusiones. 7. Bibliografía.

\section{Introducción}

¿A qué nos referimos con las expresiones expandir y profundizar la democracia? La tensión dialéctica que se produce al intentar hacer realidad los presupuestos normativos en la práctica política abre muchas áreas de conflicto en términos teóricos. Tanto en el funcionamiento de las instituciones como en la relación entre quienes toman las decisiones y el resto de la ciudadanía son múltiples los ámbitos en los que investigar la adecuación a los principios programáticos. Aquí nos centraremos en uno: el lugar que ocupan los ciudadanos en la vida pública como aspecto central en la cristalización del supuesto del "poder del pueblo" demo-cratos. El grado y las formas de inserción de los individuos en (o la relación con) la actividad política son indicadores de la calidad democrática de cualquier régimen. Ciertamente, es necesario reconocer la complejidad de la cuestión para no caer en interpretaciones simplistas que minimicen la trascendencia del tema o intenten justificar algunas deficiencias en pos de una supuesta eficacia técnica.

Planteo esta cuestión desde una perspectiva procesual y siguiendo el ejemplo de Tilly (2007) opto por pensar en "democratización en proceso" más que en democracia, enfocando los objetos de análisis y los resultados como indicadores de un fluir de avances hacia la democratización y retrocesos en forma de des-democratización. La pregunta central de investigación es la siguiente: ¿Consiguen los proyectos institucionales de participación formar ciudadanos, sujetos políticos? La pregunta subsumida en ésta será si realmente lo pretenden, pero ésta será motivo de otro texto.

Lo que denominamos sociedad civil, ciudadanía, se compone de individuos que desempeñan un rol: el de sujeto político, el de ciudadano (más o menos activo) que se responsabiliza de parte de lo público, actor necesario del juego democrático. Este sujeto político es el resultado de unas estructuras, unas circunstancias y una historia, es, por tanto, una construcción social y política. Sin dejar de considerar los condicionamientos estructurales, actuar como ciudadano es consecuencia de un aprendizaje que capacita para ejercer derechos y proceder en lo común. Este aprendizaje acontece en diversos escenarios y a través de procesos complejos de intersección entre iniciativas conscientes y voluntarias de los ciudadanos y medidas de inclusión/exclusión de las instituciones. Este artículo analiza el potencial de capacitación política de unos proyectos participativos diseñados, puestos en marcha y controlados por las instituciones políticoadministrativas en las últimas décadas. Vamos a investigar si favorecen la formación política de los participantes en términos democráticos, lo que nos permitirá valorar si, al menos en este punto, son ciertamente democratizadores como pretenden. Teniendo en cuenta la desigualdad estructural de partida de los ciudadanos a la hora de defender derechos y gestionar sus intereses, la creación de instituciones públicas que capaciten a cualquier ciudadano para la acción política -más allá de su ubicación socioeconómica 
y cultural- es una cuestión particularmente relevante por sus efectos potencialmente niveladores.

El artículo comienza mostrando a qué nos referimos con el término "proyectos institucionales de participación", explicando su sustento teórico y su relevancia de cara al tema que nos ocupa: la capacitación política. El objetivo es saber si haber formado parte de estos procesos participativos cualifica para comportarse como ciudadano activo, como sujeto político, si aporta motivación hacia lo público, profundización (o creación) de voluntad política y/o pericia y habilidades para ejercerla. Para ello aplico los resultados de una investigación propia y divido la variable dependiente en dimensiones -que presento como indicadores de capacitación política- y que formulo como preguntas de investigación. Tras una breve presentación del estudio empírico mostraré qué proyectos contribuyen a la formación de sujetos políticos y por qué. Y para comprender mejor esta dimensión formativa termino comparándola con la de otras formas participativas que desarrollan los individuos de manera espontánea.

\section{1. ¿Pretenden/pueden los proyectos institucionales de participación formar sujetos políticos?}

La delegación de la acción política para todo tipo de actividades y en todos los ámbitos, restringiendo la participación ciudadana a la elección periódica de representantes, es cada vez más discutida en tribunas públicas, cátedras universitarias y, muy claramente, en las bases sociales que cada día estrenan nuevas fórmulas reclamando mayor protagonismo e influencia contrastable en las decisiones políticas. Esta corriente participativa no es novedad en el ámbito científico y tampoco lo es en los hábitos sociales, pero ha ganado centralidad en las últimas décadas. A esto se suman, desde finales del siglo XX, los peligros que suponen para la legitimidad y la credibilidad del sistema democrático la desafección creciente y la denuncia del alejamiento de los núcleos de poder de las necesidades sociales. Problemas de coherencia normativa, cuestionamientos de la legitimidad, dificultades de integración social, incluso la previsión de conflictos que amenacen la estabilidad como consecuencia de la diminución de la justicia social, llevaron a que las propias instituciones iniciaran procedimientos de apertura.

Y así, estimulados por el empuje de intelectuales e investigadores y por el ambiente de expectativas creciente se pusieron en marcha diversos mecanismos con una -al menos teórica- intención de ampliar la participación: Presupuestos Participativos, Jurados Ciudadanos, Encuentros Ciudadanos, Encuestas Deliberativas,.... ${ }^{1}$ Todos basculan en torno a la idea de que una mayor implicación en la gestión pública es posible (y conveniente) sin que ello suponga debilitar la necesaria eficacia de la misma. Cuáles sean las instituciones que los habiliten, quiénes los sujetos protagonistas, los tiempos y maneras de implicación, los diseños procedimentales (aplicados al diagnóstico, a la toma

${ }^{1}$ Para una explicación de los proyectos de participación institucional y sus rasgos distintivos, ver Font, 2001. 
de decisiones o la implementación de las políticas,...) depende de posicionamientos ideológicos tanto como de razones de tipo instrumental. En unos casos predomina el carácter de experimento y la presencia de académicos e investigadores es central tanto en el diseño como en la gestión (Jurados Ciudadanos, Encuestas Deliberativas, Encuentros participativos) $)^{2}$. En otros el carácter es más político y práctico, se vinculan a la vida cotidiana de los participantes y se desarrollan más como experiencias que como casos de estudio (Presupuestos Participativos).

El sustrato teórico de estos mecanismos de participación se construye en torno a dos ejes: de un lado, el de la democracia participativa ${ }^{3}$ (Pateman, 1970; Barber, 1984) y su supuesto de cualificación universal según el cual todo ciudadano tiene "capacidad" para participar en la gestión de sus derechos e intereses. De otro, el de las teorías de la democracia deliberativa. La puesta en práctica de la democracia participativa tropieza con un hecho determinante: la desigualdad social que evidencia un déficit de partida para unos y una situación de privilegio para otros, lo que pretende resolver la virtud niveladora de la deliberación. La teoría de la deliberación subraya el valor de la información: una ciudadanía informada es una ciudadanía preparada para actuar, y dado que dicha información es un bien susceptible de distribuir se puede democratizar. Junto a ello esta teoría señala el valor de la deliberación, procedente de la teoría de la acción comunicativa (Habermas, 1996) que señala que reflexionar en conjunto, el ejercicio del agora, cualifica y activa a los sujetos que interactúan en unas condiciones determinadas (Fishkin, 1997; Elster, 1998). Bajo estos dos supuestos (información + deliberación) sería posible ampliar la participación a personas sin formación ni experiencia previa.

Los defensores de estos proyectos participativos señalan que el resultado de los mismos es el estímulo de la cooperación, del compromiso cívico y una gestión inteligente de la heterogeneidad que, al tiempo que "hacen real" la democracia, facilitan la integración social (Pateman, 1970) y socializan en valores de responsabilidad política y de conciencia colectiva (Barber, 1984). Barber plantea la vitalización y repolitización de contextos micro-locales y ensalza la figura del "vecino" como prototipo de sujeto político. Otros autores (Smith y Tolbert, 2004) señalan cómo transforman "los modos de la política" pasando las relaciones políticas a ocupar un lugar central en los ámbitos sociales (Baiocchi, 2005). La línea más crítica señala problemas de eficacia y plantea que sustituir el conocimiento experto y la información exhaustiva por el sentido común y la información incompleta, cuando no deficiente, resulta de dudosa utilidad. Sin embargo, según algunas experiencias la participación no sólo no está reñida con la eficacia sino que puede ser mayor en algunas materias, como la redistribución de la riqueza y la diminución de la desigualdad, por la posibilidad de adoptar, con más

\footnotetext{
${ }^{2}$ Proyectos no permanentes o mini-publicprojects
}

${ }^{3}$ Esta línea de pensamiento tiene antecedentes que se remontan a la Grecia clásica y a pensadores posteriores como Rousseau, Stuart Mill, Tocqueville, o en tiempos más recientes el propio Paulo Freire. 
facilidad, políticas públicas que favorezcan a los menos formados e informados (y más desfavorecidos), como muestra Baiocchi en el caso de Porto Alegre (2005).

El efecto educativo es uno de los más reputados y cada autor destaca unos aspectos: una ciudadanía informada produce ciudadanos con preferencias claras y bien definidas (Dahl, 1989); los ciudadanos aprenden a ajustar sus preferencias a las condiciones del entorno, lo que ayuda a la creación de vínculos comunitarios, criterios de bien común y sentido de identidad colectiva (Barber, 1984; Fishkin, 2009); aumentan la tolerancia y el respeto por opiniones ajenas y por los valores democráticos (Barber, 1984; Pateman, 1970; Habermas, 1996); desarrolllan habilidades para las prácticas políticas y la actuación en las instituciones cívicas (Pateman, 1970; Mansbridge, 1999). Incluso, algunos autores señalan que las consecuencias se extienden más allá de los participantes directos a familiares, vecinos y a los diversos actores (asociaciones, movimientos sociales) creando una nueva esfera pública (Baiocchi, 2005). Su presentación como escuelas de democracia sería una de las expresiones más gráficas de estos efectos (Talpin, 2011). Sin embargo, otros estudios plantean dudas de que en la práctica generen estos resultados, salvo en condiciones muy concretas como las del Brasil de los 80 y 90 del siglo XX. En esa estela de cuestionamiento se ubica este estudio.

\section{2. ¿Qué queremos saber?}

Queremos saber si haber formado parte de estos procesos participativos cualifica/ capacita para comportarse como ciudadanos activos, tal como señalan las teorías citadas. Es decir, si aporta motivación hacia lo público, profundización (o creación) de voluntad política, pericia y habilidades para ejercerla, etc. Concretando, las preguntas de investigación son las siguientes:

\section{Los sujetos que participan en estos procesos:}

1. ¿Desarrollan valores en relación con la solidaridad, la tolerancia y/o el bien común que estimulen la voluntad o la conciencia política?

2. ¿Adquieren conocimientos que faciliten su comprensión de la acción política y del funcionamiento de las administraciones públicas?

3. ¿Desarrollan habilidades para las relaciones sociales necesarias en la gestión de lo público?

4. ¿Adquieren destrezas para el desempeño de las tareas correspondientes?

5. ¿Generan sentimientos de identidad hacia lo colectivo, lo comunitario?

6. ¿Aumentan o disminuyen la confianza en las instituciones políticas y administrativas y/o la inclinación a colaborar en las mismas?

Estas preguntas de investigación cubren las dimensiones centrales de la capacitación política: valorativa, cognitiva, relacional, instrumental, expresiva, identitaria y evaluativa. Para contestarlas utilizaré los resultados de una investigación en la que comparamos proyectos participativos institucionales en tres países del sur de Europa: 
Italia, España y Francia ${ }^{4}$. Estudiamos: 1) su expansión; 2) sus rasgos característicos; 3) las causas que explican su puesta en marcha y 4) si producen efectos culturales en los implicados. En este texto voy a profundizar en el cuarto aspecto, y más allá de los resultados publicados en Funes, Talpin y Rull (2014) me centro aquí sólo en los efectos educativos de cara a la acción pública y la participación política. En el punto 5 del artículo retomaré y contestaré cada una de estas preguntas explotando los resultados obtenidos en dicho estudio.

Mediante una estrategia de recogida de información compuesta por búsquedas en Internet, análisis documental, encuestas por países, estudios de contexto por comunidades y explotación de datos secundarios, estudiamos los tres primeros aspectos. Las consecuencias culturales se analizaron mediante la técnica del estudio de caso aplicada a cinco proyectos (ver Tabla 1). En cada caso se llevaron a cabo 12 entrevistas en profundidad y un grupo de discusión (Funes, Talpin y Rull, 2014). Los dos criterios principales que guiaron la selección de los cinco estudios de caso fueron:

1) Asegurar una variabilidad suficiente que permitiera evaluar la potencialidad formativa de distintos modelos.

2) Identificar tipos de proyectos y tipos de participantes en los que fueran más previsibles las consecuencias buscadas. Es decir, maximizar las posibilidades de contrastar la variable dependiente extremando sus condiciones óptimas de desarrollo.

En cuanto al primer criterio las variables consideradas fueron: 1) Duración, frecuencia e intensidad: experiencias largas, con encuentros periódicos e intensidad variable (Presupuesto Participativo) y experiencias cortas e intensas que no se repiten (por ejemplo, una Encuesta Deliberativa); 2) Estado del proyecto: en marcha o terminado, para estudiar la estabilidad de los impactos; 3) Carácter consultivo o decisorio: cuando las decisiones alcanzadas son vinculantes para las autoridades (Presupuesto Participativo) y cuando "pueden" ser tenidas en cuenta (o no) (Jurados Ciudadanos, Encuestas Deliberativas); 4) Temáticas a tratar, vinculadas a la vida cotidiana de los participantes y elegidas por ellos (Presupuestos Participativos) o cuestiones de interés general pero lejanas y seleccionadas por las autoridades (Encuestas Deliberativas o Encuentros Ciudadanos); Y, por último, 5) Forma de acceso a los proyectos, los ciudadanos acuden espontáneamente (Presupuesto Participativo) o proceden de una selección muestral (Encuesta Deliberativa o Jurados Ciudadanos).

Aplicando estas variables seleccionamos los siguientes casos: 1. Presupuesto Participativo en España (Getafe, Comunidad de Madrid); 2. Presupuesto Participativo en Francia (Poitou-Charentes) en un centro de Enseñanza Secundaria. 3. Jurados Ciudadanos sobre la política del agua en Andalucía en España (que tuvieron lugar en Málaga, Almería y Jerez); 4. Encuentro Ciudadano sobre cambio climático en Francia (Poitou-Charentes) y 5. Proyecto Participativo dedicado al transporte público en España (Sabiñánigo, en Aragón).

${ }^{4}$ Proyecto de Investigación CSO2009-08968, dirigido por Joan Font, realizado entre 2010 y 2012. Para resultados véase: Font, Della Porta y Sintommer (2014). 
En cuanto al segundo criterio, maximizar las posibilidades de contrastar la variable dependiente extremando sus condiciones óptimas de desarrollo, se elaboró una muestra de participantes con distintos niveles de implicación (de lo que cabía esperar diferentes grados de afectación) para estudiar la relación entre nivel de compromiso, grado de afectación y tipo de experiencia. Se buscó el diseño que, a priori, parecía más favorable para evaluar las "potencialidades" de estos mecanismos cuando funcionan en condiciones óptimas y comparar sus resultados con los de otros modelos. Para ello el seleccionado fue el Presupuesto Participativo de Getafe, concretamente unos colectivos formados por los más implicados en el proceso que denominaron Mesas del Presupuesto Participativo.

Tabla 1: Casos de estudio seleccionados

\begin{tabular}{|c|c|c|c|c|c|}
\hline CASOS & $\begin{array}{l}\text { Duración/ } \\
\text { Frecuencia/ } \\
\text { Intensidad }\end{array}$ & $\begin{array}{c}\text { En marcha / } \\
\text { Terminado }\end{array}$ & $\begin{array}{c}\text { Consultivo / } \\
\text { Decisorio }\end{array}$ & $\begin{array}{l}\text { Temáticas: } \\
\text { elegidas/ } \\
\text { próximas - } \\
\text { impuestas/ } \\
\text { ajenas }\end{array}$ & $\begin{array}{c}\text { Adscripción } \\
\text { voluntaria / } \\
\text { Selección } \\
\text { aleatoria }\end{array}$ \\
\hline $\begin{array}{l}\text { Presupuesto } \\
\text { Participativo } \\
\text { Getafe } \\
\text { (España) }\end{array}$ & $\begin{array}{l}\text { Largo / } \\
\text { periódico / } \\
\text { intensidad } \\
\text { variable }\end{array}$ & En marcha & Decisorio & $\begin{array}{l}\text { Elegidas/ } \\
\text { próximas }\end{array}$ & Voluntaria \\
\hline $\begin{array}{l}\text { Presupuesto } \\
\text { Participativo } \\
\text { (Francia) }\end{array}$ & $\begin{array}{l}\text { Largo / } \\
\text { periódico / } \\
\text { intensidad } \\
\text { variable }\end{array}$ & En marcha & Decisorio & $\begin{array}{l}\text { Elegidas/ } \\
\text { próximas }\end{array}$ & Voluntaria \\
\hline $\begin{array}{l}\text { Jurados } \\
\text { Ciudadanos } \\
\text { Andalucía } \\
\text { (España) }\end{array}$ & $\begin{array}{l}\text { Corto / } \\
\text { intenso }\end{array}$ & $\begin{array}{l}\text { Dos años } \\
\text { atrás }\end{array}$ & Consultivo & $\begin{array}{l}\text { Impuestas / } \\
\text { ajenas }\end{array}$ & $\begin{array}{l}\text { Selección } \\
\text { aleatoria }\end{array}$ \\
\hline $\begin{array}{l}\text { Encuentro } \\
\text { Ciudadano } \\
\text { (Francia) }\end{array}$ & $\begin{array}{l}\text { Corto / } \\
\text { intenso }\end{array}$ & $\begin{array}{l}\text { Más de un } \\
\text { año antes }\end{array}$ & Consultivo & $\begin{array}{l}\text { Impuestas / } \\
\text { ajenas }\end{array}$ & $\begin{array}{l}\text { Selección } \\
\text { aleatoria }\end{array}$ \\
\hline $\begin{array}{l}\text { Proyecto } \\
\text { Participativo } \\
\text { Sabiñánigo } \\
\text { (España) }\end{array}$ & $\begin{array}{l}\text { Corto / } \\
\text { intenso }\end{array}$ & $\begin{array}{l}\text { Más de un } \\
\text { año antes }\end{array}$ & Consultivo & $\begin{array}{l}\text { Impuestas / } \\
\text { próximas }\end{array}$ & Voluntaria \\
\hline
\end{tabular}

Fuente: Elaboración propia. 


\section{Revisando lo que ya sabemos,,..$y$ yendo más allá ${ }^{5}$}

\subsection{Constatación de efectos limitados y condicionados}

El estudio confirmó la potencialidad formativa de los procesos pero también que ésta es relativa y condicionada. Cerca del $50 \%$ de los entrevistados afirman que la influencia recibida y/o mantenida en el tiempo es nula. Sólo se consiguen efectos formativos bajo determinadas circunstancias, principalmente procedimentales pero también de tipo individual. Es más, en ocasiones se logra lo contrario de lo pretendido, más desafección, menos ilusión y confianza en la política, en las instituciones y en su implicación cívica. Clasificamos los efectos en cuatro tipos: cognitivos (conocimientos que adquieren); expresivos (sentimientos que generan); instrumentales (habilidades y destrezas que aprenden); y evaluativos (valoraciones y evaluaciones que transforman). El efecto más extendido, en más sujetos y en todo tipo de experimentos, es el cognitivo. Aprenden en relación con la materia del proceso (la política del agua, el cambio climático, el transporte público,...) o en relación con el ámbito en que tiene lugar (administración local, autonómica,...) y los conocimientos son relativamente duraderos en una mayoría de participantes. Efectos expresivos, evaluativos e instrumentales se encontraron en menos de la mitad. Distinguiendo entre la extensión y la intensidad del impacto el efecto cognitivo es el más extendido pero no el más intenso, y no tiene el valor transformador del expresivo que, siendo minoritario, es intenso y produce cambios estables. Los instrumentales son muy limitados en los proyectos no permanentes (minipublic projects) pero claros y transformadores en los permanentes; y los expresivos son casi inexistentes en los no permanentes. Efectos instrumentales y expresivos sólo están claros en los Presupuestos Participativos y los evaluativos, intensos aunque más minoritarios, también en los no permanentes.

\subsection{Variables explicativas de los efectos contrastados}

¿A qué se deben los efectos señalados? Puesto que queremos investigar las posibilidades formativas de los mecanismos de participación, obviamos la información relativa a otros factores explicativos y nos centramos en las variables del diseño ${ }^{6}$.

\subsubsection{Longitud y frecuencia de los encuentros}

Como vimos en el apartado teórico la democracia se aprende "practicando" y el aprendizaje necesita repetición y duración para convertir las experiencias en hábitos y evitar que se diluyan (Eliasoph, 2011). Ello explicaría que los Presupuestos Participativos que reúnen estas dos condiciones produzcan más resultados. Los Jurados Ciudadanos, las Encuestas Deliberativas,... duran un día, dos a lo sumo. Los participantes se muestran muy impresionados (gratamente impresionados) cuando cumplimentan la encuesta de control al finalizar el acto pero este efecto se diluye con el paso del tiempo,

${ }^{5}$ Para ver los resultados de forma más extensa ver Funes, Talpin y Rull (2014).

${ }^{6}$ En Funes, Talpin y Rull (2014) se analizan otras variables independientes explicativas de tipo individual y relacional que no afectan al objeto de este artículo. 
al menos, si no hay un seguimiento ${ }^{7}$. Un factor adicional que intensifica el efecto de estas dos variables es que la repetición y la estabilidad de los encuentros permite crear relaciones, redes sociales que se sostienen en esta experiencia y que, con independencia de si se mantienen o no en la vida cotidiana, refuerzan los cambios de cada uno y fortalecen el vínculo con el proyecto creando un nuevo ámbito afectivo y político del que los participantes sienten que forman parte. Ello corrobora la importancia del factor tiempo que señalan los estudios de Eliasoph (2011).

\subsubsection{Proximidad de los temas tratados}

El segundo aspecto significativo es la relación de las temáticas que se discuten con la vida cotidiana de los participantes. La generación de vínculos de identificación con los asuntos y entre los participantes (vecinos, compañeros), genera más implicación y compromiso. Por ejemplo, la necesidad de un semáforo en un cruce peligroso motivará el seguimiento del proceso, para conocer el éxito o fracaso de la propuesta, más que el cambio climático o la política del agua que resultan cuestiones más lejanas. También influye si son los participantes o las autoridades quienes deciden los temas. Si se trata de atender un problema percibido por los vecinos al que se quiere dar solución la implicación será mayor, mayor la satisfacción si se consigue y más consistentes los efectos. Además, y de nuevo, trabajar juntos en algo de cuya resolución obtendrán beneficio todos, o resolverán algún problema común, intensifica los lazos entre los afectados que pueden dar lugar a posteriores desarrollos participativos.

\subsubsection{Intensidad de la implicación y asunción de responsabilidades}

Cuando existen órganos intermedios entre la asamblea general de ciudadanos y la administración -y los participantes gozan de un cierto grado de autonomía- los efectos formativos se intensifican, dado que los sujetos asumen alguna responsabilidad sobre la viabilidad del proyecto. La evidencia la encontramos en las Mesas del Presupuesto de Getafe donde los miembros desarrollaban iniciativas, incluso modificaron algunas reglas de funcionamiento, lo que favoreció que asumieran cierto protagonismo ${ }^{8}$. El impacto educativo se multiplica pero, ciertamente, se reduce el número de los beneficiados, ya que son muchos menos los que participan en las Mesas que en la asamblea general. A mayor intensidad de impacto menor expansión del mismo. ¿Podemos pensar que la capacidad formativa de estos grupos intermedios del Presupuesto Participativo es equivalente a la de los pequeños grupos de debate de proyectos como los Jurados

\footnotetext{
${ }^{7}$ En los proyectos no permanentes los participantes comienzan rellenando un cuestionario y terminan rellenando el mismo cuestionario para detectar los cambios producidos. Al no hacer un seguimiento posterior los primeros estudios magnificaron los cambios conseguidos, al dar por válidos los reflejados en el formulario conclusivo del experimento. En Ganuza et al. (2012) se añade un cuestionario en un tercer momento (seis meses después) y se comprueba que el efecto se ha diluido en gran medida. Fishkin (2009) en una toma de contacto diez meses después afirma que bastantes cambios se mantenían. Nuestro estudio dejó pasar más tiempo y nuestra interpretación es que son poco duraderos.
}

${ }^{8}$ Entrevista $n^{\mathrm{o}}$ 1, en Funes, Talpin y Rull, 2014: 176. 
Ciudadanos? La respuesta es no; la potencialidad formativa no es tan sólo cuestión del número de personas en interacción. Los pequeños grupos de los Jurados Ciudadanos, por seguir con el ejemplo, eran de cinco o seis personas por lo que la interacción era fácil e intensa; pero debatían sobre un tema seleccionado por la autoridad convocante, según un guion pautado y controlado por el facilitador; y sus opciones eran dialogar, responder preguntas y consensuar acuerdos guiados. En cambio, en las Mesas del Presupuesto Participativo de Getafe los vecinos convocaban cuando querían para deliberar sobre lo que ellos decidían. La autonomía podría explicar el mayor impacto de unas experiencias frente a otras más que el número de implicados ${ }^{9} i$ Sería ésta la solución al dilema que plantea "a más participación menos deliberación y viceversa" al que llegan tantos estudios? (Font, Della Porta y Sintommer, 2014:203). ¿Evitaría tener que elegir entre una o la otra? Parece que sí, que la autonomía en la gestión resulta una variable determinante en este sentido.

\subsubsection{Carácter decisorio de los proyectos}

En los proyectos no vinculantes: Encuentros Ciudadanos, Jurados Ciudadanos, Encuestas Deliberativas los participantes no tienen siempre claro cuál es su papel, ni si influirán en las políticas públicas o no las conclusiones de los eventos. Sin embargo, en los Presupuestos Participativos los implicados tienen garantizado que decidirán el gasto de un porcentaje del presupuesto municipal. El aspecto decisorio tiene consecuencias en términos formativos dado que comprobar que el esfuerzo y el tiempo dedicados influyen en las decisiones públicas produce empoderamiento.

\section{4. ...Y yendo más allá: ¿construyen sujetos políticos?}

¿Podemos decir que los efectos detectados conforman el rol de ciudadano, de sujeto político activo? Para ello retomo las preguntas de investigación que planteé como dimensiones de capacitación política al inicio del artículo. El sujeto político respondería a un perfil valorativo en relación con el bien común, la solidaridad, la tolerancia (pregunta 1); un sentido de la eficacia personal, sentimiento de eficacia política interna ${ }^{10}$, entendido como la confianza en la capacidad individual para afrontar lo colectivo (preguntas 2, 3, 4); un sentimiento de identificación con lo colectivo: comunidad, vecindario, pueblo,... (pregunta 5); y confianza en las instituciones, sentimiento de eficacia política externa (pregunta 6).

\subsection{Los valores: conciencia política y sentido del bien común}

Pregunta 1: Los sujetos que participan en estos proyectos ¿desarrollan valores en relación con la solidaridad, la tolerancia, el bien común, que animen una voluntad política?

${ }^{9}$ En este sentido véase también, Lang (2010).

${ }^{10}$ El término eficacia política procede de Campbell, Gurin y Miller (1954) más tarde se divide en dos dimensiones: interna y externa (Craig, Niemi y Silver, 1990). 
Encontrar cambios valorativos en experiencias que no se repiten y cuya duración es como máximo de dos días es poco probable, por lo que prestamos más atención a los proyectos permanentes. En ellos los participantes que tenían experiencia previa en asociaciones, partidos o movimientos sociales (o simplemente un discurso ideológico definido) consideraban que generar valores en relación con el bien común era un objetivo irrenunciable; pero se cumplía escasamente. En cambio, los implicados con un perfil ideológico más neutro y sin experiencia en acciones colectivas no consideraban este aspecto como un posible (o deseable) resultado. Cuando la participación es instrumental y el principal objetivo es solucionar un problema personal no se produce este cambio de valores. Por tanto, participar no conduce, necesariamente, a cambios valorativos en este sentido.

Sin embargo, todos los entrevistados, incluidos los de los proyectos cortos, valoraban haber tenido la oportunidad de conocer a personas con problemas y circunstancias distintas, a las que en su vida diaria difícilmente hubieran encontrado. Apreciaban que el ejercicio de la deliberación les permitió ponerse en el lugar del otro, lo que calificaban como ejercicio de "apertura de mente" ${ }^{11}$. Otro resultado generalizado era la valoración del carácter democrático de estas experiencias, frente al déficit en actores políticos convencionales e instituciones, y la conveniencia de que se multiplicaran para que impactaran en un ámbito político más amplio. Asimismo, se detectaba mayor sensibilización en relación con temas concretos, por ejemplo, en el JJCC sobre el agua y en el proyecto francés sobre el cambio climático ${ }^{12}$. Lo que no queda claro es que esto se pueda catalogar como cambio de valores.

¿Supone, por tanto, la experiencia participativa, un avance en la construcción de una idea de bien común, de sensibilización hacia lo colectivo, de solidaridad y tolerancia, tal como señalan los estudios de Pateman (1970) y Barber (1984)? Encontramos una gradación: desde una minoría que cambió incluso los hábitos personales desarrollando un comportamiento empático ${ }^{13}$; hasta quienes más allá de la sorpresa inicial no concretaban ningún cambio en valores ni actitudes. La respuesta sería que el cambio es limitado, pero los que ya presentaban rasgos de solidaridad, tolerancia o de una voluntad política incipiente los enriquecían. Znaniezky (1992) nos enseñaba que los cambios siguen la secuencia que va de la tendencia (más general) a la actitud (formulación personal) y de esta a la actividad (prácticas concretas); éste sería el cambio valorativo cuando tenía lugar. Más que generar valores inexistentes reforzaba los que estaban en ciernes. Pero no más. De hecho, los más comprometidos mostraban su desilusión al comprobar que la mayoría utilizaba estos mecanismos para obtener beneficios para sí mismos o sus más próximos sin que movilizara conciencias ni generara valores

${ }^{11}$ Este efecto es contrastado también por los estudios de Pinnington and Schugurensky (2010).

${ }^{12}$ Ejemplos: entrevistados en Encuentro Ciudadano francés $n^{\circ} 2$ y 5, Proyecto Participativo en Aragón, E. nº 3; GD de los JJCC, (Funes, Talpin y Rull, 2014: 166-7).

${ }^{13}$ Ejemplos Entrevistas $n^{\circ} 2,4$ y 7 del Presupuesto Participativo español, véanse datos en ibid: 221. 
nuevos ${ }^{14}$. En este sentido, aunque no podemos confirmar los resultados más negativos de Kuklinski et al. (1993) que plantea que la solidaridad o empatía pueden incluso disminuir, ni los más optimistas de Pateman (1970) y Barber (1984), nos situamos en una posición intermedia, cercana a Mutz (2006: 79-84), que avala el aumento de estas virtudes cuando los antecedentes lo hacen previsible.

\subsection{Empoderamiento: autoconfianza y capacitación para la política}

Pregunta 2: Los individuos que participan en estos proyectos iadquieren conocimientos que facilitan su comprensión de la acción política y/o del funcionamiento de las administraciones públicas?

La respuesta mayoritaria es afirmativa en los proyectos permanentes y mínima en los no permanentes. En los Presupuestos Participativos de Getafe todos, y en el de Poitou-Charentes casi todos, valoran el conocimiento adquirido sobre las instituciones. Además, quienes más se implicaban entablaban contacto con responsables políticos y administrativos, aprendían el funcionamiento de los procedimientos, las legislaciones pertinentes y también las dificultades burocráticas (a medida que las propuestas ya seleccionadas avanzaban por la retahíla de despachos en busca de firmas y permisos). En las experiencias breves hablar de "aprendizaje" puede resultar sobredimensionado, pero adquieren conocimientos sobre procedimientos, normativas, límites en los que actuar, actores implicados; aunque pasado un tiempo son asuntos en gran medida olvidados. Tanto unos como otros aprenden sobre las temáticas que tratan ${ }^{15}$.

El grado de conocimiento adquirido (y/o mantenido) depende de muchas variables: de la calidad y compromiso de los facilitadores; de la implicación de los especialistas que presentan los informes; también de la voluntad política de las instituciones que si están interesadas en la formación (e implicación) de los participantes comunicarán las decisiones tomadas y explicarán si su aportación fue tenida en cuenta, o no; lo que animará al seguimiento de la participación o (en su defecto) provocará desánimo ${ }^{16}$. Pero depende también de los propios participantes. Los más entusiastas aprenden más y lo consideran valioso. Los más escépticos, bien por falta de interés, por desconfianza en las instituciones, o porque sólo les guía una motivación instrumental que se consume en lo inmediato (por ejemplo, la asignación económica que en algunos casos reciben por su participación en el proyecto), manifiestan que no aprendieron nada significativo en el sentido que nos ocupa (la capacitación política) ni en ningún otro.

${ }^{14}$ Comentarios de Entrevistas no 2, 3 y 5 del Presupuesto Participativo español, ibid, 221.

${ }^{15}$ Ejemplos: GD de JJCC en España; Encuentro Ciudadano francés, Entrevista ${ }^{\circ} 2$ y 5 ; Proyecto Participativo aragonés Entrevista no 3, ibid: 166-7; 221-2.

${ }^{16}$ Nienmeyer (2011) también resalta la importancia de la forma de comunicar los resultados. 
Pregunta 3: Los ciudadanos que participan ¿desarrollan habilidades para las relaciones sociales necesarias en la gestión de lo público?

Aquí la diferencia es significativa entre proyectos permanentes y no permanentes. En los Presupuestos Participativos sí desarrollan, en mayor o menor medida en función del grado de implicación, habilidades relacionales quienes carecían de ellas. Asistir a reuniones, atender asambleas, participar en los debates, y, sobre todo, programar y preparar los encuentros, informar a los vecinos de las propuestas aceptadas, de la evolución de las ejecuciones, ... todas estas actividades, que desarrollan al menos los miembros de las Mesas del Presupuesto Participativo de Getafe, generan habilidades relacionales y amplían las redes sociales lo que genera recursos estratégicos, y confirma las afirmaciones de Pateman (1970) y Mansbridge (1999). A esto se añade la posibilidad de contactar con responsables políticos, alcaldes, concejales, el arquitecto del ayuntamiento que explica la viabilidad o las dificultades de un proyecto urbanístico puesto en marcha; la dirección del centro educativo para discutir la aplicación de una propuesta aprobada para mejorar la escuela,.... La posibilidad de interactuar con las autoridades mejora la autovaloración a nivel relacional. Aquí la variable que resulta más importante de las destacadas es la vinculación de los participantes a las temáticas ( $\sin$ obviar, evidentemente, el efecto de las otras tres). Las relaciones se establecen con más facilidad cuando hay interés personal en los asuntos y ello refuerza la comunicación con personas no implicadas pero afectadas, otros vecinos del mismo barrio, por ejemplo. Los participantes ven cómo el lugar que ocupan en su comunidad se transforma: ellos ejercen un nuevo rol, se dirigen a los vecinos desde una posición de conocimiento y su entorno les reconoce ese lugar, les preguntan, les piden que promuevan una acción u otra, ... ${ }^{17}$

Pregunta 4: Los ciudadanos que participan ¿adquieren habilidades para el desempeño de actividades y tareas públicas?

Actuar como un ciudadano activo requiere un grado de confianza y autovaloración tan necesarios o más que los conocimientos o los contactos con responsables políticos. Habilidades como el hablar en público, redactar documentos, desenvolverse en los diversos frentes de la Administración, presentar y defender argumentos, movilizar recursos materiales y humanos,... son destrezas que, más allá de su valor práctico favorecen la autoconfianza y la seguridad personal. Es lo que en ciencia política se denomina eficacia política interna, la conciencia de la capacidad personal para enfrentar y atender las cuestiones políticas. Aun cuando el nivel educativo suele estar relacionado con muchas de estas capacidades no lo está siempre de igual modo y hay otras variables de corte individual que considerar. El estudio de Fraile (2014) muestra el género como condicionante de primer orden. Las mujeres tienen más dificultades para algunas

${ }^{17}$ Ejemplos: Entrevistas $n^{\mathrm{o}}$ 1, 2, 8 del Presupuesto Participativo del caso español (Funes, Talpin y Rull 2014: 176,221). 
de estas tareas, aunque parecen ser más expertas en otras ${ }^{18}$. Mujer, mayor, con bajo nivel educativo y sin experiencia asociativa previa $^{19}$ es el perfil que presenta menor capacidad para la acción política. Los entrevistados valoraban los cambios producidos en estas mujeres de más edad, a las que decían estimular y apoyar, y ellas manifestaban su satisfacción al ver cómo adquirían recursos que no tenían ${ }^{20}$.

Pero no sólo ellas. Jóvenes, mayores, hombres y mujeres valoran la posibilidad de exponer opiniones, de ser escuchados, y se reconocen el mérito de haber sido capaces de expresarse, de argumentar y hacer valer sus propuestas gracias a estas experiencias ${ }^{21}$. La percepción subjetiva de capacidad es un efecto comprobado; en los proyectos permanentes por las facilidades que da la recurrencia y persistencia de los actos, y en los no permanentes, aunque en menor grado, porque el facilitador interviene para asegurar igualdad de oportunidades para intervenir. También se aprende a escuchar, a ponerse en el lugar del otro, al menos, durante el tiempo que dura la reunión. Discutir sobre argumentos dispares en un debate controlado y orientado por expertos promueve una escucha respetuosa en prácticas de tolerancia. En este sentido la experiencia deliberativa es educativa en todos los proyectos analizados. Mutz (2006) subraya cómo el diseño institucional del marco deliberativo facilita la predisposición a una escucha más atenta de la que se da en la vida cotidiana.

En los Presupuestos Participativos los aprendizajes son claros tanto en la dimensión expresiva como en la instrumental. Tener que competir con otros vecinos o compañeros para conseguir que la asamblea apruebe la opción deseada estimula la creatividad. Dos jóvenes de Getafe, sin ninguna experiencia asociativa previa, desarrollaron habilidades para reclutar apoyos en favor de su propuesta (la creación de un parque para perros); explican sus estrategias para movilizar a sus vecinos y conseguir que se aprobara su propuesta: "Parecía un político haciendo campaña" 22. Lo que aprendieron les llevó, posteriormente, a crear una asociación al efecto. $\mathrm{O}$ el caso de un estudiante francés que relataba cómo la formación adquirida le dotó de habilidades para la acción política, hasta el punto de continuar como representante primero en su área geográfica y posteriormente en la Unión Europea. El impacto educativo que supone asumir responsabilidades en la gestión de estos proyectos es valorado también en otros estudios Lang (2010), Baiocchi y Ganuza (2014).

${ }^{18}$ Funes (1995) muestra la mayor capacidad en este sector social para unos tipos de gestión interna y menos recursos para la expresión pública.

${ }^{19}$ La participación como elemento de compensación de niveles bajos de educación formal se analiza en Funes (2006) y en cuanto a las mujeres de más edad ver Funes (2011).

${ }^{20}$ Entrevistas $\mathrm{n}^{\mathrm{o}} 2$ y 8 del Presupuesto Participativo de Getafe, (Funes, Talpin, Rull, 2014:176).

${ }^{21}$ Iguales resultados se encuentran en DelliCaprini et al. (2004), Talpin (2011) y Funes (2006). La eficacia política interna se encuentra como resultado también en Morrell (2005).

${ }^{22}$ Entrevista ${ }^{\circ} 4$; Entrevistas $n^{\circ} 4$ y 7 del Presupuesto Participativo en Getafe y Entrevista $\mathrm{n}^{\mathrm{o}} 8$ del Presupuesto Participativo del caso francés (Funes, Talpin y Rull 2014: 171-3; 221-2). 


\subsection{Creando "comunidad" y sentido de identidad colectiva}

Pregunta 5: Los ciudadanos que participan igeneran sentimientos de identidad con (y responsabilidad hacia) lo colectivo, lo comunitario?

Sólo encontramos este efecto en los proyectos que implican capacidad decisoria y que tienen un enraizamiento, identificación territorial (un barrio, una localidad) o identificación con un proyecto como es el caso del centro educativo en el Presupuesto Participativo francés. Ratificamos lo que señalaba Baiocchi (2005) en la experiencia de Porto Alegre, la sensación de ser vecino es poderosa (siguiendo a Barber, 1984) y es indicador del sujeto político, en tanto que categoría incluyente. El sentimiento de identidad es consecuencia (a veces también causa) de cooperar con quienes se comparte intereses y beneficios potenciales: si se arregla la calle nos beneficiamos todos, si continúa embarrada todos salimos perjudicados; el hecho de compartir tanto el problema como la solución genera y refuerza identidad. Además, implicarse en el ámbito de convivencia marca diferencias con quien no lo hace, construye la (auto)imagen de "persona responsable"que se preocupa por el bien común, sentimiento con importantes efectos de empoderamiento. Al tiempo, añade el reconocimiento de los demás, los que te conocen y te encuentran en las actividades cotidianas te devuelven esa percepción de persona comprometida, "saben que tú estás detrás" de aquel semáforo que se cambió de lugar o de la pista de patinaje para los chavales,... ${ }^{23} \mathrm{El}$ reconocimiento supone satisfacción y la satisfacción refuerza esa identidad: Como decía una participante de Getafe con entusiasmo: "Otros tienen "títulos" dicen yo soy tal o cual. Yo no, yo soy "VECINA", eso "VECINA" ${ }^{24}$, lo que refuerza lo señalado por Barber (1984). Aunque este efecto tiene una relación directa con la intensidad de la implicación, y, por lo tanto, dependerá de si se trabaja mucho o poco, desde el punto de vista de las potencialidades del proyecto es altamente dependiente de las temáticas trabajadas, sobre todo de si están incardinadas en el ámbito de proximidad. La visibilización de los éxitos es un factor consistente con el refuerzo del empoderamiento y de la identidad colectiva.

\subsection{Confianza (o desconfianza) en las instituciones}

Pregunta 6: Los ciudadanos que participan ¿aumentan o disminuyen su confianza en las instituciones políticas y administrativas y la inclinación a colaborar en las mismas?

En términos de eficacia política externa hallamos resultados afirmativos y negativos. En unos casos la cercanía a las instituciones provoca la identificación con los políticos o los responsables administrativos y genera, si no confianza al menos comprensión ante las dificultades de la burocracia, la lentitud y las complejidades que la resolución de los

${ }^{23}$ Recordemos la interpretación de Mead (1999), desde el interaccionismos simbólico, sobre la construcción del yo en referencia a la figura del "otro generalizado".

${ }^{24}$ Entrevista $^{\circ}{ }^{\circ}$. Otros ejemplos: Entrevistas 2, 5 y 8 del PP en Getafe, ibid: 176 y 221. 
problemas entraña ${ }^{25}$. Pero esta misma cercanía incrementa la exigencia y las críticas. Lo que parece incuestionable es que adquieren elementos de diagnóstico para evaluar la acción política que antes no tenían. Analizar esta dimensión desvela un importante punto de riesgo de estos proyectos: las expectativas creadas y el ajuste de las mismas a la realidad. Crear expectativas estimula la participación; pero defraudarlas puede causar desilusión y rechazo. Hemos encontrado personas que multiplicaron su escepticismo, o incluso su visión negativa de las instituciones después de la experiencia participativa. Mostramos dos casos.

En el primero la sensación era, en buena medida, de engaño y manipulación. Los miembros de los Jurados Ciudadanos fueron individualmente seleccionados, convocados y se pidió su colaboración; la Junta de Andalucía se dirigió a ellos como representantes de los intereses de los andaluces considerando importantes sus opiniones que, según se les indicó, serían contempladas en la nueva política sobre el agua. Este reclamo y este tratamiento inicial generaron empoderamiento y motivaron la implicación. Sin embargo, terminado el acto nadie les informó si los acuerdos a los que llegaron fueron tenidos en cuenta o completamente ignorados, ni de qué medidas fueron finalmente adoptadas. Pasaron de sentirse como personas "con autoridad y criterio" a verse como ciudadanos normales, completamente ignorados. Uno de los participantes del Grupo de Discusión llegaba al extremo de plantear el proceso como simulación intencionada de participación, cuyo objetivo real era legitimar medidas impopulares (la Junta de Andalucía aumentó las cargas impositivas relacionadas con el acceso al agua) justificándolas en la "opinión de los ciudadanos consultados". La comunicación posterior, el qué y cómo se informa, y su vinculación a cuestiones significativas para los ciudadanos es relevante en estos mini-public projects, como señala, también, Niemeyer $(2011)^{26}$.

El segundo caso lo encontramos en el Presupuesto Participativo español. Getafe se divide en siete barrios y cada uno gestionaba su porcentaje del presupuesto. En uno de ellos se aprobó la creación de un parque para mayores y la ejecución se prolongó durante años; en ese tiempo el ayuntamiento decidió implantar la misma medida en otro barrio y se desarrolló mucho antes que en el distrito que lo propuso y aprobó en Presupuesto Participativo. Es decir, unos vecinos trabajaron, defendieron, movilizaron... y ganaron, su propuesta fue aprobada pero tardó en ser puesta en marcha. Los vecinos de otro distrito sin ningún esfuerzo y en menos tiempo pudieron disfrutarla. ¿Cuál es la clave? los procedimientos municipales regulares fueron más ágiles que los del Presupuesto Participativo que fueron largos y engorrosos; tal vez, la voluntad política fue diferente en un caso y en otro. La pregunta que se hacía la persona que lo planteó era ¿merece la pena el esfuerzo participativo si, en definitiva, sólo importa que un político tome la

${ }^{25}$ Entrevistas $n^{\circ}, 3$ y 5 del Presupuesto Participativo francés y Entrevistas $n^{\circ} 1,2$ y 8 del Presupuesto Participativo de Getafe (Funes, Talpin y Rull, 2014: 221,2).

${ }^{26}$ Con las mismas razones explicaban el aumento de desconfianza tras los procesos Talpin y Wojcik (2010). 
decisión de ejecutar una idea? ${ }^{27}$ La voluntad política de las instituciones, su capacidad técnica y económica evidencian si priorizan (o infravaloran) el proceso; y ello tiene consecuencias en la desafección o el apego a la participación, y, en definitiva, en la motivación o el desinterés que provocan hacia lo político.

Más allá de estos dos casos, aunque muchos expresan satisfacción, que hacen extensible a otros ámbitos de la vida política, otros manifiestan descontento ${ }^{28}$. Este descontento se debía a lo restrictivo de los procesos: porque no podían elegir los motivos de los debates ni controlar sus resultados; o porque las temáticas sobre las que podían decidir eran poco relevantes. La sensación de ser parte de un juego, de una simulación, aun no siendo general sí era significativa. Además, si los procesos participativos no están dotados de medios, personal y normativas facilitadoras que eviten que su puesta en marcha se alargue en exceso, o que sean frenados por las dificultades burocráticas, un potencial efecto positivo se puede convertir en negativo con el consiguiente aumento de la desconfianza. Y, por último, si se pretende generar empoderamiento mostrando una imagen activa del ciudadano en las decisiones públicas conviene considerar el efecto que puede producir no atender lo ofertado. Generar más expectativas de las que se pueda (o se pretenda) atender puede aumentar la desconfianza y la desafección.

\section{Comparando con otras formas de construir ciudadanos}

A pesar de las luces, las sombras y las grandes diferencias entre ellos, parece que estos proyectos pueden capacitar para la acción política y formar ciudadanos competentes, motivados y comprometidos, si bien con matices y de manera condicionada. Hemos visto las características que favorecen el impacto educativo y la mejor combinación de las mismas. Llegados hasta aquí cabe preguntarse por las similitudes y diferencias entre el potencial de capacitación política de estos proyectos y el de otros de base social. Es decir, si es más fácil (o más probable) formarse como sujeto político en una asociación de vecinos o en una organización en defensa de la Sanidad Pública, que en un Presupuesto Participativo. No podemos olvidar que poner en marcha mecanismos de participación institucional supone costes económicos para las arcas públicas por lo que parece conveniente evaluar la utilidad de estos desembolsos ${ }^{29}$. La pregunta sería: en términos formativos ¿aportan alguna singularidad estos proyectos frente a otros en los que está comprobado que se adquieren las capacidades políticas buscadas? ¿Debería tenerse en cuenta lo contrastado como efectivo en el mundo asociativo para mejorar la capacidad formativa de los proyectos institucionales? Ciertamente, no pretendo mostrar

${ }^{27}$ Entrevista ${ }^{\circ}$ 10, Presupuesto Participativo de Getafe, (Funes, Talpin y Rull, 2014: 170 y 221).

${ }^{28}$ Entrevistas $n^{0} 1$ y 11 del Presupuesto Participativo francés, véase datos en ibid: 221-2. En el mismo sentido en Talpin (2011) y Font y Navarro (2013), entre otros.

${ }^{29}$ Estos proyectos institucionales abarcan más objetivos que la capacitación de los participantes pero aquí se evalúa esta dimensión. 
las potencialidades formativas de las asociaciones ${ }^{30}$, a lo que dediqué estudios previos (Funes, 2011; 2006; 2003; 1998 y 1995), sino utilizar dichos conocimientos para afinar los resultados expuestos.

En cuanto a la promoción de valores o principios éticos (pregunta 1) tanto en las asociaciones como en los proyectos institucionales se trata de una oportunidad relativa más que de una consecuencia clara. La participación asociativa es consecuencia de poseer unos determinados valores más que causante de los mismos, pero permite desarrollarlos cuando estos son "latentes no practicados", en cuyo caso, al igual que los proyectos participativos, las asociaciones brindan la oportunidad de pasar de la tendencia a la actitud, de ésta a la acción. En este sentido el tipo de asociación es variable condicionante ${ }^{31}$, las asociaciones de heteroayuda y de tipo político convocan a personas con unos valores de solidaridad o servicio público, o provocan su desarrollo; no ocurre en las de ocio o tiempo libre más que de manera anecdótica. Sin embargo, los proyectos institucionales tienen más probabilidades de promover valores de tolerancia y solidaridad cuando no existen que las asociaciones, debido a la mayor homogeneidad de miembros en las segundas y la presumible mayor heterogeneidad en los primeros. Es decir, dado que las asociaciones tienen perfiles muy definidos acuden a ellas personas con valores similares, mientras que en los proyectos institucionales la mezcla de perfiles, sobre todo cuando la selección procede de un muestreo aleatorio, es más común. Si en estos casos la intervención técnica gestiona adecuadamente esta diversidad puede favorecer (como pudimos comprobar en el estudio realizado) valores de tolerancia donde antes no existían.

En cambio, en cuanto a la adquisición de eficacia política interna -capacidades cognitivas, relacionales e instrumentales- (preguntas 2,3 y 4), puesto que el efecto está directamente relacionado con el tiempo de exposición, la diferencia entre asociaciones y proyectos institucionales es importante y a favor de las primeras, dada la ausencia de continuidad de la mayoría de los proyectos institucionales (y teniendo en cuenta que ésta, cuando existe, depende de la voluntad política). Ahora bien, lo que hay que resaltar es que desarrollar estas capacidades no tiene que ver tanto con el mecanismo institucional o el tipo de asociación como con el tipo de participación que se practica y el lugar que cada individuo ocupa en el colectivo (o en el proyecto). Tanto da buscar recursos para una verbena donde reclutar fondos para una asociación de barrio; como gestionar permisos para organizar unas jornadas informativas en un movimiento social; conseguir aliados para incrementar la presión sobre las autoridades ante un cambio legislativo que se estima lesivo; o desde un sindicato preparar la defensa de un compañero expedientado...en todas estas actividades se adquieren conocimientos, se establecen relaciones y se desarrollan destrezas y habilidades de tipo instrumental que cualifican a los individuos como sujetos políticos (Funes, 2006). Ciertamente, la asunción de este

${ }^{30}$ Toda una línea de pensamiento corrobora la dimensión educativa de las asociaciones en términos democráticos; destacan Pateman (1970), van Deth (1997) y Putnam (2000), entre muchos otros.

${ }^{31}$ Para más información sobre otros factores véase Funes (1995 y 2006) 
tipo de tareas es más frecuente en una asociación que en un proyecto institucional. Incluso cuando éstos favorecen la implicación de los participantes la probabilidad de ejercer estas prácticas es menor debido a la tutela de la institución promotora, lo que no ocurre en las asociaciones. Es, por tanto, una potencialidad formativa muy extendida en las asociaciones, debido a la autonomía con que actúan los participantes, y muy limitada en los proyectos institucionales.

Ahora bien, en relación con algunas habilidades expresivas como: la adquisición de seguridad y confianza para hablar en público, defender ideas, ejercitar una escucha respetuosa y tolerante... los proyectos institucionales presentan alguna ventaja. En los casos estudiados comprobamos que la deliberación tutelada estimula a todos los participantes intentando que nadie deje de beneficiarse en términos de desarrollo personal. Al tratarse de experiencias con un importante grado de artificio es posible disminuir los efectos de la desigualdad de partida, favoreciendo que todos tomen parte activa en el proceso y puedan adquirir no sólo capacidad sino autoconfianza. Sin embargo, esta función educativa puede verse minimizada por el carácter ocasional y discontinuo de los encuentros. Asimismo, hacer pivotar sobre los técnicos la capacitación en los términos referidos supone depender de su formación y motivación, lo que implica un grado de aleatoriedad a tener en cuenta.

Por otra parte, en relación con la dimensión identitaria (pregunta 5) dos aspectos resaltan a favor de los proyectos institucionales. Los efectos en términos de identidad que conseguía el Presupuesto Participativo de Getafe respondían, en buena medida, a su dimensión decisoria y vinculante, algo que no se da en las asociaciones (aun cuando los éxitos, cuando se obtienen, cumplan la misma función). El reconocimiento que obtenían de sus vecinos se aseguraba por la "visibilización" de los éxitos, ya que la identificación entre proyecto/activista/barrio se retroalimentaba en las relaciones de proximidad (cada vecino sabía que su vecino participante había ayudado a la consecución: del arreglo de una plaza, la instalación de un semáforo en un cruce peligroso,...). Por otro lado, en las asociaciones el refuerzo de la identidad con el colectivo puede resultar problemático. Todas generan vínculos de identificación (entre miembros, con el proyecto, con el lugar,...) de hecho este tipo de vínculos afectivos son uno de los recursos más eficaces para compensar los costes de la implicación (Medina y Ramiro, 2006:298). Ahora bien, éstos no siempre son consistentes con valores democráticos, en cuyo caso no pueden considerarse educativos en los términos estipulados. Las asociaciones pueden cimentar valores cívicos o estimular el enfrentamiento y la exclusión (Funes, 1998) ${ }^{32}$; algo difícil de imaginar en los proyectos institucionales que, por su propio formato tutelado, o no generan vínculos identitarios (por la temporalidad o fragilidad de los proyectos) o son de carácter inclusivo.

${ }^{32}$ En Funes (1998) se estudian las consecuencias de vincularse a identidades colectivas en conflicto con el entorno social, centrando la reflexión en los sectores violentos de la izquierda abertzale en los años noventa del siglo XX. 


\section{Conclusiones}

¿Pueden capacitar estos proyectos institucionales para la acción política? A grandes rasgos sí pero de manera muy diferente según los diseños. Sólo de manera limitada generan voluntad política o valores en relación con el bien común; sí permiten que afloren cuando son latentes y los enriquecen si son incipientes. Pueden generar valores de solidaridad y tolerancia cuando la gestión técnica del proyecto trabaja con éxito la heterogeneidad de los miembros, pero no deja de ser un aspecto particularmente difícil y dependiente de la capacidad del personal técnico a cargo y, en todo caso, de la duración del proyecto. La adquisición de conocimientos, habilidades y destrezas instrumentales está también condicionada al tiempo de exposición, extremadamente corto en los proyectos no permanentes y en los permanentes se da si se asume responsabilidad en la gestión con cierta autonomía. Cuando hay una relación continuada entre participantes y autoridades, una dedicación intensa y relevante por parte de los participantes, y se obtienen éxitos sustantivos gracias a la virtualidad decisoria de los proyectos, se consigue cualificación individual, y ésta se intensifica con el reconocimiento del entorno que prospera en términos de capital social. Sin embargo, es dudosa la producción de eficacia política externa, dado que los datos muestran que los sujetos pueden reaccionar ante las debilidades de los proyectos convirtiendo la percepción de una atención insuficiente, o los incumplimientos de las expectativas generadas, en distancia y crítica de las instituciones que, además, se generalizan al ámbito general de la política.

¿Por qué comparar con los efectos educativos de la participación asociativa? Porque identificar los aspectos en los que éstas son pedagógicamente válidas ayudaría a replicarlos en los proyectos institucionales, pero sumándole dos ventajas de las propuestas institucionales. La primera que al estar vinculadas a decisiones públicas efectivas su impacto formativo se multiplica, ya que significa incidencia real en la vida de los participantes y su entorno, y en ámbitos pequeños eso supone un reconocimiento social que enriquece aún más la autoconfianza y el compromiso con lo público. La segunda, que permite incorporar a quienes menos recursos relacionales, culturales, materiales y operativos poseen -ya que es más difícil que éstos por propia iniciativa den el paso de implicarse en una asociación- incidiendo así en la nivelación de capacidades.

$\mathrm{La}$ artificialidad de los proyectos es tanto su punto fuerte como su punto débil. Por ejemplo, que personal experto dirija y controle la deliberación favorece la capacitación de quienes más carecen de ella (quienes más la necesitan), lo que puede disminuir la desigualdad social de partida. Pero en la medida en que sea determinante la voluntad política de la autoridad de turno los proyectos serán dependientes de la disponibilidad de medios económicos y técnicos para ofrecer asistencia apropiada. Voluntad política que asegure continuidad en el tiempo, carácter vinculante de las decisiones y una atención técnica para lograr una difusión que motive a los más alejados de la práctica participativa, son los puntos que permiten a estas instituciones ampliar las competencias políticas. Hay que tener en cuenta que la atención técnica transforma, en alguna medida, el funcionamiento de las instituciones, ya que implica integrar a los ciudadanos en una parte de su trabajo cotidiano y ello puede suponer dificultades o resistencias internas. Por todo ello, la pretendida consecuencia formativa requiere por parte de las instituciones comprometerse con el proyecto por sus valores democráticos 
y no por su valor estético susceptible de rendimientos electorales a corto plazo, y recordar que implantar proyectos sin la suficiente cobertura (por este orden): política, presupuestaria y técnica, puede producir efectos perversos.

Pero, en definitiva, lo determinante sería conocer los objetivos reales de estos proyectos. Si importa la función educativa democratizadora o si, por el contario, no pasan de ser políticas de imagen y legitimación de la gestión. Si su meta se reduce a facilitar la gobernanza no se dirigen a un ciudadano como sujeto político sino como usuario/cliente/consumidor $\mathrm{y}$, en ese caso, la potencialidad educativa habría que evaluarla de manera distinta ${ }^{33}$.

\section{Bibliografía}

Baiocchi, G. (2005): Militants and Citizens. The Politics of Participatory Democracy in Porto Alegre, Stanford, Stanford University Press.

Baiocchi, G. y E. Ganuza (2014): "Participatory Budget as if emancipation mattered", Politics and Society, 42 (1), pp. 29-50.

Barber, B. (1984): Strong Democracy, Participatory Politics for a New Age, Berkeley, University of California Press.

Campbell, A., G. Gurin y W. E. Miller (1954): The Voter Decides, Evanston IL, Row, Peterson.

Colino, C. y E. del Pino (2008): "Democracia participativa en el nivel local: debates y experiencias en Europa", Revista catalana de dret públic, 37, pp. 247-283.

Craig, S. C., R. Niemi y G. Sliver (1990): "Political Efficacy and Trust: a report on the NES pilot study items", Political Behaviour, 12, pp. 289-314.

Dahl, R. (1989): Democracy and Its Critics, New Haven Conn., Yale University Press.

Delli Carpini, M.X., F.X. Cook y L.R. Jacobs (2004): "Public Deliberation, Discursive Participation, and Citizen Engagement", Annual Review of Political Science, 7, pp. 315-344.

Eliasoph, N. (2011): Making Volunteers. Civic Life after Welfare's End, Princeton, Princeton University Press.

Elster, J. (1998): “Deliberation and Constitution making”, en J. Ester (ed.), Deliberative Democracy, Cambridge, Cambridge University Press.

Fishkin, J. (1997): The Voice of the People, New Haven, Yale University.

Fishkin, J. (2009): When the People Speak. Deliberative Democracy and Public Consultation, New York, Oxford University Press.

Font, J. (ed.) (2001): Ciudadanos y decisiones públicas, Barcelona, Ariel.

Font, J. y C. Navarro (2013): "Personal Experience and the Evaluation of Participatory Instruments in Spanish Cities", Public Administration, 91 (3), pp. 616-631.

${ }^{33}$ Colino y del Pino (2008) reflexionan sobre si los procesos participativos buscan "auténtica" participación o si atienden otros objetivos no explicitados. En este sentido, Baiocchi y Ganuza (2014) reflejan el deterioro y devaluación en términos democratizadores del proyecto más potente de todos, el Presupuesto Participativo. 
Font, J., D. Della Porta e Y. Sintomer (2014): Participatory Democracy in Sothern Europe: Causes, characteristics and consequences, Londres, Rowman and Littlefield.

Fraile, M. (2014): "Does deliberation contribute to decreasing the gender gap in knolewdge?" European Union Politics, 15 (3), pp. 372-388.

Funes, M.J. (1995): La ilusión solidaria, Madrid, Universidad Nacional de Educación a Distancia.

Funes, M.J. (1998): "Social responses to political violence. A case of study: Social Movements and their Audience", Journal of Conflict Resolution, 42 (4), pp. 403-510.

Funes, M.J. (2003): "Socialización política y participación ciudadana: jóvenes en dictadura y jóvenes en democracia", en Jóvenes, Constitución y cultura democrática. Revista de Estudios de Juventud, Edición Especial 25 años de la Constitución Española, pp. 57-76.

Funes, M.J. (2006): “La experiencia de la acción colectiva”, en J.R. Montero, J. Font y M. Torcal, eds., Ciudadanos, asociaciones y participación en España, Madrid, Centro de Investigaciones Sociológicas, pp. 301-322.

Funes, M.J. (2011): “La participación en asociaciones de la población mayor de sesenta y cinco años en España: análisis de sus efectos e indicaciones para las políticas públicas sectoriales", Revista Internacional de Sociología, 69 (1), pp. 167-193.

Funes, M.J., J. Talpin y M. Rull (2014): "The cultural consequences of engagement in participatory process", en J. Font, D. Della Porta e Y. Sintomer (eds.), Participatory Democracy in Southern Europe: Causes, characteristics and consequences, Londres, Rowman and Littlefield, pp. 151-190.

Ganuza, E., F. Francés, R. Lafuente y F. Garrido (2012): "Do Participants Change Their Preferences in Deliberative Processes", Revista Española de Investigaciones Sociológicas, 139, pp. 225-246.

Habermas, J. (1996): Between Facts and Norms: Contributions to a Discourse Theory of Law and Democracy, Cambridge, The MIT Press.

Kuklinski, J.H., E. Riggle, V. Ottati, N. Schwarz y R.S. Wyer (1993): "Thinking about Political Tolerance, More or Less, with More or Less Information", en G.E. Marcus y R. Hanom (eds.), Reconsidering the Democratic Public, University Park, Pennsilvania State University Press, pp. 225-248.

Lang, A. (2010): "Citizen Learning in State-Sponsored Institutions. Accounting for variation in the British Columbia and Ontario Citizens' Assemblies on Electoral Reform", en E. Pinnington y D. Schugurensky (eds.), Learning Citizenship by Practicing Democracy: International Initiatives and Perspectives, Newcastle upon Tyne, Cambridge Scholars Publishing, pp. 168-185.

Mansbridge, J. (1999): "On the idea that participation makes better citizens", en S.

Elkin y K. Soltan (eds.), Citizen Competence and Democratic Institutions, Philadelphia, The Pennsylvania University Press, pp. 291-325.

Mead, G.H. (1999): Espiritu, Persona y Sociedad, México, Paidós.

Medina, L. y L. Ramiro (2006): "La participación en las asociaciones: actitudes y estructuras organizativas", en J.R. Montero, J. Font y M. Torcal (eds.), Ciudadanos, asociaciones y participación en España, Madrid, Centro de Investigaciones Sociológicas, pp. 281-300. 
Morrell, M. E. (2005): "Deliberation, Democratic Decision-Making and Internal Political Efficacy", Political Behavior, 27 (1), pp. 49-69.

Mutz, D. C. (2006): Hearing the other side. Deliberative vs. Participatory Democracy, Cambridge, Cambridge University Press.

Niemeyer, S. (2011): "The Emancipatory Effect of Deliberation Empirical. Lessons from Mini-Publics", Politics and Society, 39, pp. 103-140.

Pateman, C. (1970): Participation and Democratic Theory, Cambridge, Cambridge University Press.

Pinnington, E. y D. Schugurensky (2010): “Civic Learning and Political Engagement through Participatory Budgeting: The Case of Guelph, Canada", en E. Pinnington y D. Schugurensky (eds.), Learning Citizenship by Practicing Democracy: International Initiatives and Perspectives, Newcastle upon Tyne, Cambridge Scholars Publishing, pp. 286-305.

Putnam, R. D. (2000): Bowling Alone: The Collapse and Revival of American Community, New York, Simon and Schuster.

Smith, D. A. y C. J. Tolbert (2004): Educated by Initiative: the effects of direct democracy on citizens and political organizations, Ann Arbor, University of Michigan Press.

Talpin, J. (2011): Schools of democracy. How ordinary citizens (sometimes) become competent in participatory budgeting institutions, Colchester, ECPR Press.

Talpin, J. y S. Wojcik (2010): "Deliberating environmental policy issues: Comparing the learning potential of online and face-to-face discussions on climate change", Policy \& Internet, 2 (2), pp. 61-93.

Tilly, C. (2007): Democracy, Cambridge, Cambridge University Press.

Van Deth, J. W. (1997): "Introduction. Social involvement and democratic politics", en J.W. Van Deth (ed.), Private Groups and Public Life: Social participation, voluntary associations and political involvement in representative democracies, London, Routledge.

Znaniecki, F. (1992): "Principios para la selección de datos culturales", Revista Española de Investigaciones Sociológicas, 60, pp. 153-168. 